\title{
"Gamma Anna": A classroom demonstration for teaching the concepts of Gamma Imaging
}

Nicola Wolff ${ }^{1}$, Jennifer Griffiths ${ }^{1,2}$ and Rebecca Yerworth ${ }^{1}$

1 Dept. Medical physics and Biomedical Engineering, University College London, Gower Street, WC1E 6BT

2 Faculty of Engineering Sciences, University College London, Gower Street, WC1E 6BT

Email: $\underline{\text { r.yerworth@ucl.ac.uk }}$

\section{Abstract}

Gamma Imaging is at the interface of Medicine and Physics and thus its teaching is important in both fields. Pedagogic literature highlights the benefits of interactive demonstrations in teaching: an increase in enjoyment and interest, as well as improvement in academic achievement. However Gamma imaging uses radioactive sources, which are potentially dangerous and thus their use is tightly controlled.

We have developed a demonstration which uses a localised exothermic reaction within a rag doll as an analogue of radioactivity. This can be safely used in classrooms to demonstrate the principles of Gamma imaging. The tool is easy to make, cheap, robust and portable. The supplementary material in this paper gives teacher notes and a description of how to make the rag doll demonstrator.

We have tested the tool using six participants, acting as 'teachers', who carried out the demonstration and described the doll as easy to use, and the 'tumour' clearly identifiable. The teaching tool was separately demonstrated to a group of 12 GCSE Physics students and a group of 12 Medical students. Feedback showed increased student engagement, enjoyment and understanding of gamma imaging. Previous research has shown that these benefits have an impact on learning and academic outcomes.

\section{Introduction}

Gamma imaging is a diagnostic imaging technique that uses radioactive tracers to provide important functional physiological information that other imaging modalities cannot (Radiological Society of North America, 2016). As an example of physics with a direct biological/medical application, and ethical implications, learning about Gamma imaging is relevant to several different areas in the secondary school curriculum and could be useful in encouraging students, particularly girls to study physics past GCSE level (Murphy \& Whitelegg, 2006).

The process of radioisotope detection by a gamma camera involves several complex physical principles. A radioactive substance is injected or ingested by the patient. The radioisotope itself, or the substance it is chemically attached to, is then selectively taken up into an area of the body (e.g. lodine into the thyroid or glucose into tumours - i.e. where there is rapid cell growth). The radioactive isotope emits gamma rays, which are detected by a gamma camera. The gamma camera produces a functional image of 'hot spots' that can be used to aid diagnosis (Institute of Physics, n.d.). 
Pedagogic literatures show that, in general, active learning and the use of interactive demonstrations have positive benefits on both engagement and learning. A study on increasing enrolment in Year 11 Physics showed that $61 \%$ of students found practical demonstrations to be helpful, and $78 \%$ said they were enjoyable (George \& Taylor, 2001). Also, over $70 \%$ of students would prefer demonstrations "often" or "very often" (Johnson, et al., 1998). The influence that interactive demonstrations have on enjoyment and interest has been shown to have a positive impact on academic achievement. As students play an important role in their own education, their interest and motivation has significant effects on their learning experiences and outcomes (Peyton, 1998, pp. 14-15). When students are actively involved in their education, and contemplate the information they have been given, they understand and retain information better (Bradforth, et al., 2015). Laukenmann et al. identified a positive correlation between enjoyment \& interest and competence within the topic (2003). Ainley et al. identified the strongest model for this link, which suggests that interest stimulates an emotional response (2002). This response then leads to an increased level of persistence, which directly positively impacts learning.

Owing to the radioactive substances involved, it is not safe to demonstrate real Gamma imaging in the classroom. However, we suggest that the pedagogic benefits of active leaning can still be achieved if an analogue is used. In this paper we describe the development of a classroom-friendly demonstration to explain the process of gamma imaging through the use of a thermal imaging camera and an exothermic reaction.

Several of an exothermic reaction's properties are analogues of the properties of a gamma-emitter. Therefore, an exothermic reaction can be used to mimic radioactivity without the dangers of ionising radiation being present. The heat generated by an exothermic reaction can be detected by thermal imaging cameras but not observed by the naked eye, just as gamma rays are invisible but can be detected by a gamma camera. Heat and gamma rays can both travel through materials: this means that they can be detected from outside of the body/phantom.

Our demonstration uses an exothermic reaction with two reactants; one is used to represent a tumour and, the other, the radioactive substance. In this work, the tumour representing reactant is placed in the desired location of a modified rag doll. The second reactant is a liquid introduced via a network of tubes. It is only where the two reactants meet that is heat produced. The resulting localised increase in temperature is detected using a thermal imaging camera, which represents a Gamma camera.

Two exothermal processes were tested: the absorption of water by wool (Lysine, the amino acid in wool, forms hydrogen bonds with water (Leeder, 1984)) and Plaster of Paris (2CaSO4. $1 / 2 \mathrm{H} 2 \mathrm{O}+3 \mathrm{H} 2 \mathrm{O}$ $==>2 \mathrm{CaSO} 4 \cdot 2 \mathrm{H} 2 \mathrm{O}+$ heat (Jørgensen $\&$ and Posneb, 1959)). Wool was initially preferred as it is a reversible reaction, but it was found that the rate of absorption was too slow for our requirements, for both raw Southdown fleece and Merino yarn. Two types of Plaster of Paris were tested. A minimal temperature increase was seen when water was added to $20 \mathrm{~g}$ of 'fine casting powder' (Mouldmaster), but there was a rise of several degrees with Plaster of Paris powder from Hobbycraft (www.hobbycraft.co.uk). However, the peak temperature occurred 40-50 minutes after the addition of water. Higher water temperatures were found to make no difference to this time, despite previous evidence that higher water temperatures cause more rapid hardening (Lavalette, et al., 
1982). However, adding common salt ( $\mathrm{NaCl})$ as a catalyst (Hamer \& Hamer, 2004) caused the time to peak temperature rise to reduce to $10-15$ minutes.

\section{Method}

\section{The doll}

A rag doll ${ }^{1}$ was made from lightweight washable fabric (curtain lining), with an opening at the back closed with Velcro, and a small opening for a mouth. The head was stuffed with toy filling, and the limbs with expanded polystyrene beads; the torso was left unstuffed. The doll was $74 \mathrm{~cm}$ tall, which is large enough to have multiple 'tumour' locations, but small enough to be covered by one thermal imaging camera image.

A leaky tubing network was constructed to distribute saline throughout the doll. For this, slits were cut in a loop of $6 \mathrm{~mm}$ diameter clear plastic tubing, joined with y-connectors to a short spur (Figure 1). This was inserted into the doll, with the tube opening forming the doll's 'mouth'. The doll was loosely stuffed with pieces of open weave cloth, arranged around the tube network, to ensure that all the locations where the 'tumour' could be placed would absorb saline injected through the 'mouth'.

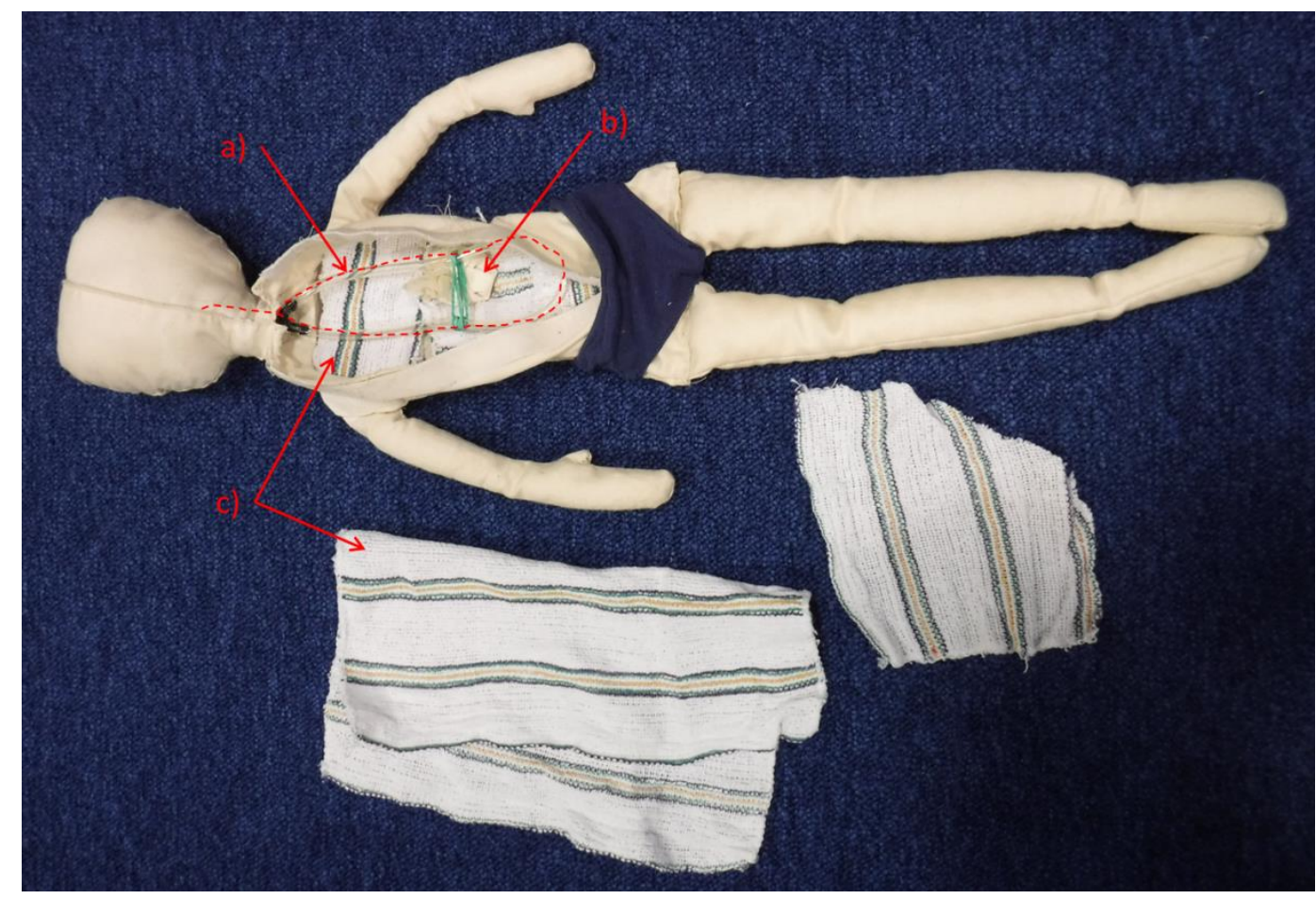

Figure 1 Back view of doll, showing internal structure: a) leaky tubing (highlighted with red dotted line), b) plaster of paris bundle (example position), c) open weave cloths

\footnotetext{
${ }^{1}$ There are many free patterns online e.g. http://jensidea.blogspot.co.uk/2011/11/rag-doll-tutorial.html.
} 


\section{The 'tumours' \& 'radioactive tracer'}

For the tumours, 20g portions of Plaster of Paris powder were weighed out and individually wrapped in squares of thin material (e.g. poly-cotton). Before each demonstration, one was placed in the doll and the back fastening closed.

A saline solution of 1 level tablespoon of salt per $150 \mathrm{ml}$ of tap water was made up, representing the radioactive tracer. A syringe was filled with $15 \mathrm{ml}$ of this solution for each demonstration.

\section{The demonstration}

A thermal imaging camera (FLIR One Personal Thermal Imaging Camera for iPhone $5 / 5 \mathrm{~S}$ ) was used to observe the doll in its initial state.

Then $15 \mathrm{ml}$ of saline were injected slowly into the 'mouth' of the doll.

The thermal imaging camera was used to record an image of the doll immediately and after 5, 10, 15 , and 20 minutes, observing when it was easiest to identify the location of the 'tumour'.

After the demonstration, the used 'tumour' was removed and discarded. The open weave cloths were also removed, and spread out on a radiator for 10-15 minutes, to dry separately from the doll.

\section{Testing}

The demonstration was piloted separately with two groups of students (group 1: 12 GCSE physics students; group 2: $122^{\text {nd }} / 3^{\text {rd }}$ year Medical students) and 6 demonstrators.

Each demonstrator tested the doll by following the provided instructions (online supplement), then filled in a usability questionnaire.

Each student group listened to a course/age appropriate PowerPoint presentation then watched the demonstration before answering questions on a work sheet (online supplement).

\section{Results}

Thermal images of the doll clearly show the location of the 'tumour' from 10 minutes after saline application (Figure 2). It was found that the clearest results were obtained when the 'tumour' was placed directly against the dolls 'skin', with the open weave cloths behind and to the sides of the tumour. 


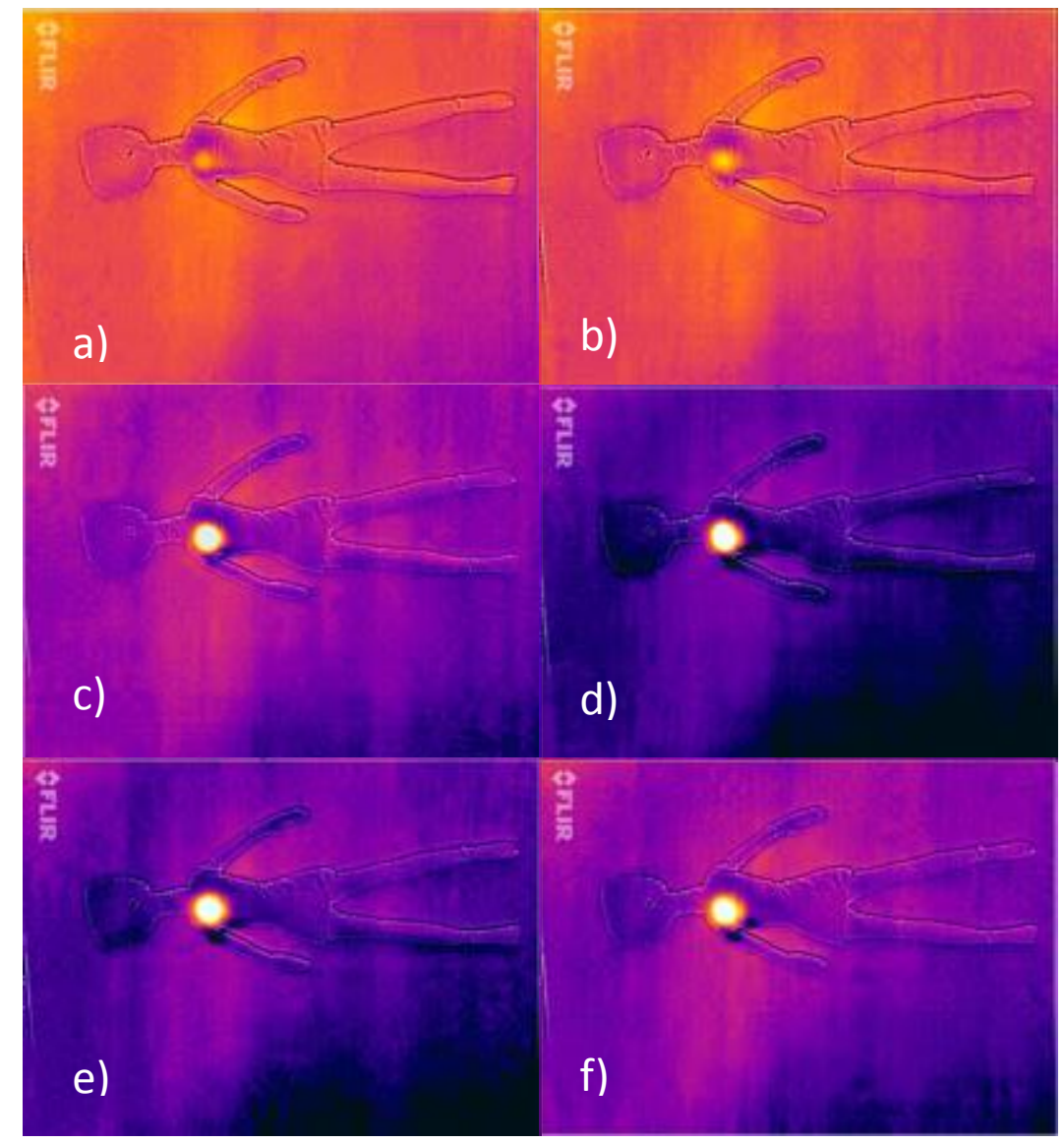

Figure 2 Thermal images of the doll a-f: 0, 5, 10, 15, 20, \& 25 minutes after saline injection. A bright spot becomes clearly visible in the right shoulder from 10 minutes, with the peak temperature rise at 15 minutes. Images were auto scaled.

Feedback from demonstrators regarding the usability of the doll was universally positive, with all 6 users rating 4 or 5 out of five for all criteria (Table 1).

Table 1 Feedback from demonstrators

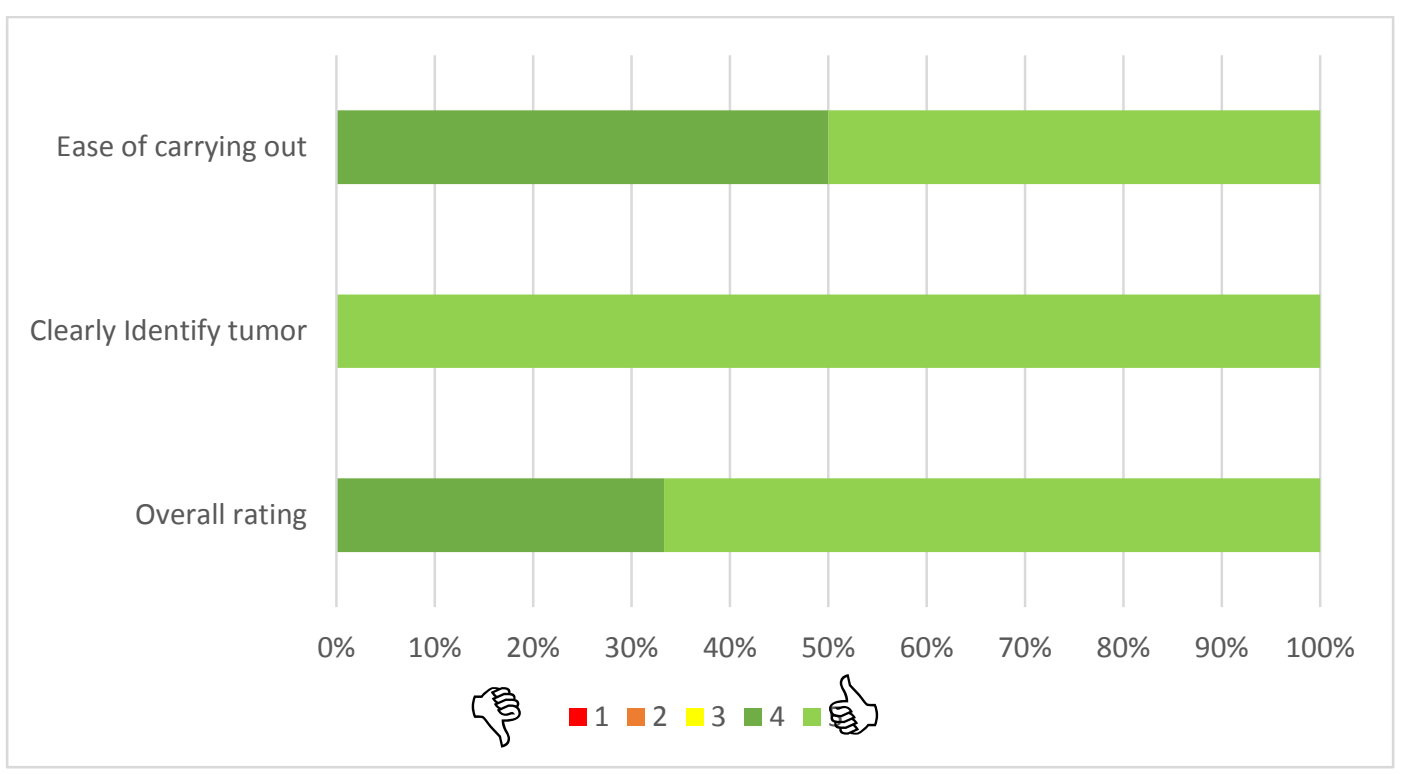

Student feedback was also positive, with at least 9 students (66\%) from each group agreeing or strongly agreeing with the questions (Table 2), and $100 \%$ for some questions. A couple of GCSE 
students were unsure if it would help them remember the topic, and the only other scores of less than 3 were one GCSE student answering how enjoyable the demonstration was, and one answering on its ability to demonstrate the selective uptake of the isotope. With respect to the worksheet, both groups answered most questions correctly, with the questions related to the effects on other people proving the most challenging.

Table 2 Student responses to questionnaire

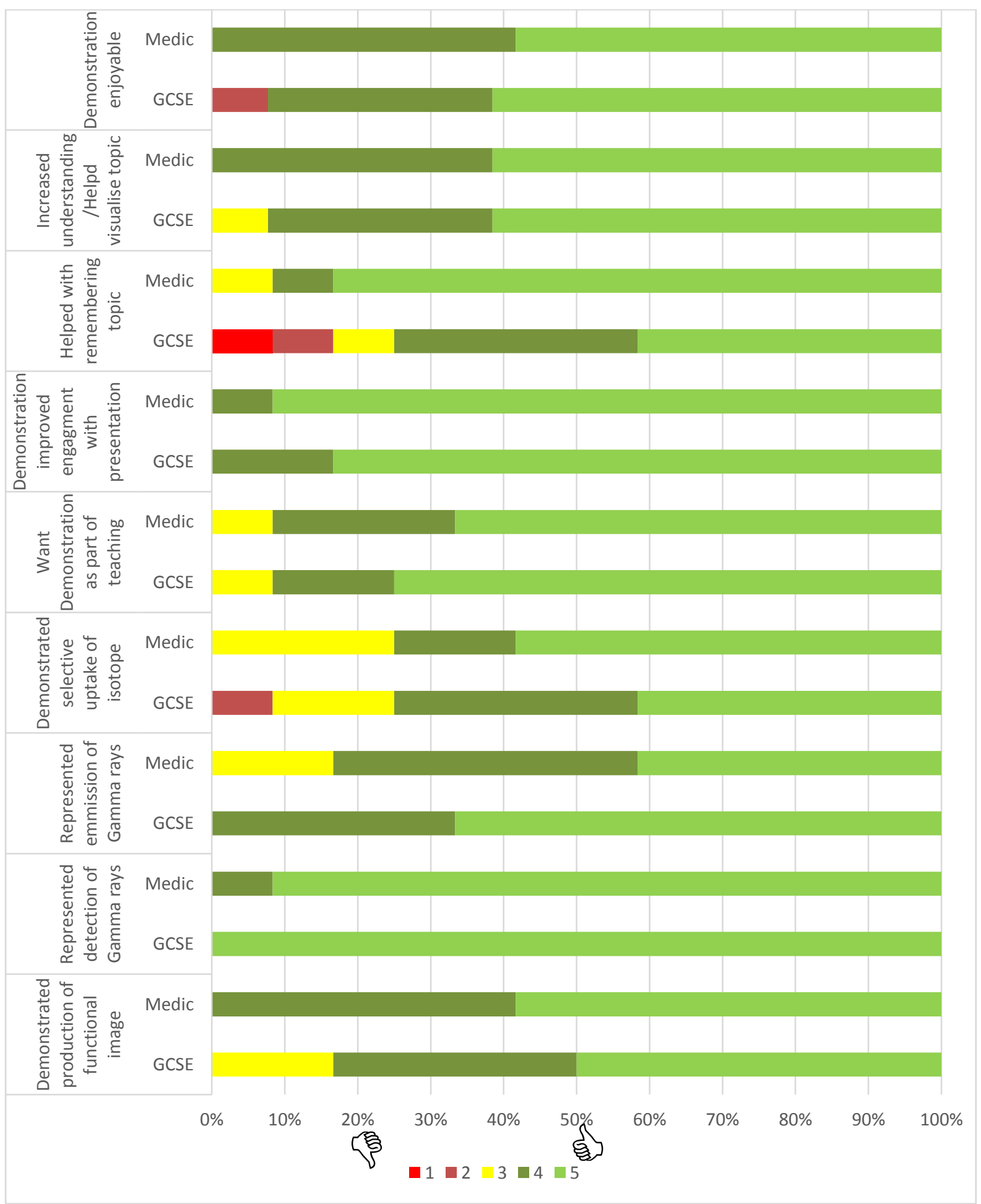




\section{Discussion}

An exothermic reaction has been identified which can be used to demonstrate the principles of gamma imaging, as used in medicine. The use of a reversible reaction, e.g. using wool and water, would have enabled repeated use of the doll with minimal maintenance. However, the issue of slow water absorption remains unsolved, and the proposed Plaster of Paris method has the advantage of allowing the stuffing to be removed for quick drying, and the location of the tumour to be varied. Feedback from all six demonstrators was positive with half of them giving the demonstration kit the highest possible score for usability and the others rating it 4/5.

The use of a cloth doll to enclose the exothermic reaction helps students to understand the medical applications of gamma imaging. The use of a two reagent system means that both tumours and radioisotope labelled medicine can be simulated, as well as the relationship between the two. At the start of the demonstration the 'tumour' (Plaster of Paris) is at an un-known location(s) within the body. The 'radioisotope' (saline) is administered to the patient; it then disperses throughout the body, but is selectively taken up by the 'tumour'. This takes time so the patient has a wait between taking the medicine and having the actual imaging scan. This too is simulated in this demonstration, albeit on a slightly a condensed timescale, so as to fit into an hour long lesson. Where this simulation differs from gamma imaging is that gamma rays are spontaneously produced by the radioisotope decaying, even whilst traveling to the target tissue. Whereas in this simulation it is a chemical reaction between the 'medicine' and the 'tumour', thus heat is only produced at the 'tumour' location. Radioisotope activity reduces exponentially as a function of time, at a rate determined by the half-life of the isotope. The temperature of the 'tumour' is a function of the current temperature and how much un-reacted plaster is in contact with water, however, since this demonstration works by comparing images at only two time points (just before saline administration and after 10-15 minutes), this is not a problem. It may, however, be useful to take a third image at the end of the lesson ( 25-30 $\mathrm{min}$ ) to illustrate that a patient will still be 'hot'/radioactive for some time after their scan, and they will need to limit their contact with other people during this time.

The use of a thermal imaging camera demonstrated clearly the detection from inside the 'body' of information which is not visible to the naked eye, though the mechanisms by which gamma cameras detect and localise gamma rays are not the same and would need separate consideration if part of the syllabus. Thermal cameras normally produce pseudo-colour images with the 'tumour' showing as a bright hot spot, as opposed to the dark regions on grey scale images produced by gamma cameras, and this could cause confusion if not addressed. A demonstration using 'grey scale' and 'negative' effect options in an imaging processing package would resolve this confusion (Figure 3). It should also be noted that real gamma images have a grainier appearance, and some normal structures may be visible - e.g. the bladder can be seen in Figure 3Figure 3A - as some healthy tissues may absorb small quantities of the radioisotope. Also, only 2-dimensional imaging has been simulated. 3dimensional gamma images may be obtained by rotating the camera around the body then reconstructing the result. Such tomographic imaging methods are not unique to gamma imaging, and we considered that it would cause confusion to include them within this demonstration. 


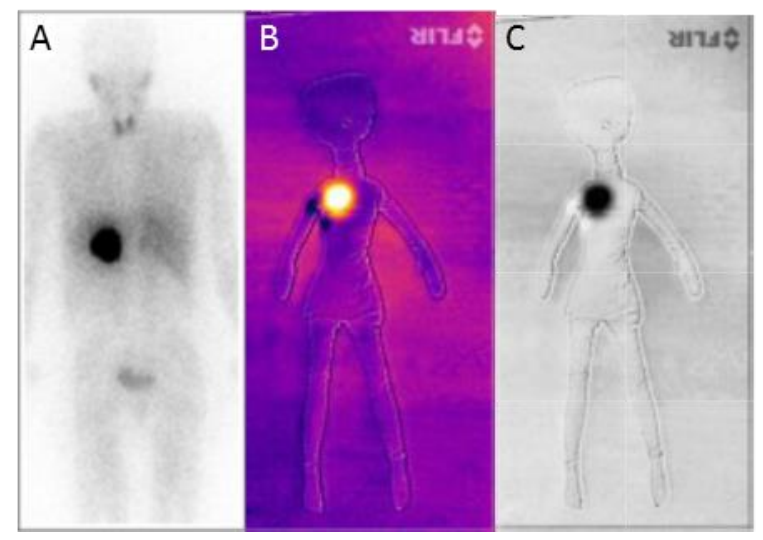

Figure 3 Simple image processing can be used to convert thermal images to more closely match Gamma Images: A) Gamma scan of patient with phechromocytoma (Drahreg01 2010), B) Thermal image of doll at 25 minutes C) as B but with grayscale and negative image effects applied.

A very large proportion of both groups of students felt that the demonstration made them more engaged, and the majority also felt that it helped them visualise or more fully understand the ideas presented in the teaching. Given the results of other pedagogic research (Bower \& Winzenz, 1970) (Carini, et al., 2006), we can be confident that the increased engagement added to the understanding provided by the visualisation, and together these will translate into better and more accurate recall of the subject.

There were four aspects of gamma imaging that were selected to be represented within the demonstration. It was found that the detection of gamma rays by the gamma camera was represented best in the demonstration, with only one student not answering "very well" when asked about its representation. The emission of gamma rays from a specific area and the production of a functional image were also represented well in the demonstration. This shows that the aims of the teaching tool were well achieved, in terms of the principles that were depicted.

While the demonstration helped to solidify these ideas for students, it expressed none of the more complex Physics within the gamma camera. To have attempted to capture all the Physics of gamma imaging within a single demonstration and lesson would likely be counterproductive, as it has also been shown that simpler demonstrations may actually be the most beneficial. Students may fail to follow a more complex demonstration, resulting in a negative impact on self-esteem, enjoyment and, therefore, motivation (Gross, 2002). Additionally, studies have indicated the importance of the analysis and context of a demonstration: Couch et al (2004)showed that without related questions or student reflection, demonstrations may be futile. Therefore, it is more important to devote time to discussing the demonstration and how its ideas are applied elsewhere than to spend time on a lengthy and complicated demonstration. Examples of the student worksheets are available (supplementary material). These can be tailored to accommodate the background understanding and learning objectives of the class. In addition to the materials used with the two student groups tested (GCSE and Medical), there is an information sheet aimed at young children needing a Gamma scan. This could be used, in conjunction with structured play using the doll, to reduce the anxiety of such patients, or it could be modified for use in schools as a general introduction to medical imaging for younger children. 
In conclusion, a safe, cheap, and easy to use demonstration of the principles of Gamma imaging has been developed and tested. When used with appropriate worksheets and introductory materials, this proved popular with students, which, according to educational theory, will improve understanding and retention of the topic.

\section{References}

Ainley, M., Hidi, S. \& Berndorff, D., 2002. Interest, learning, and the psychological processes. Journal of educational psychology, 93(3), p. 545.

Bower, G. H. \& Winzenz, D., 1970. Comparison of associative learning strategies. Psychonomic, 20(2), pp. 119-120.

Bradforth, S. E. et al., 2015. University learning: improve undergraduate science. Nature, Volume 523, pp. 282-284.

Carini, R. M., Kuh, G. D. \& Klein, S. P., 2006. Student engagement and student learning: Testing the linkages. Research in higher education, 47(1), pp. 1-32.

Crouch, C., Fagen, A. P., Callan, J. P. \& Mazur, E., 2004. Classroom demonstrations: Learning. American journal of physics, 72(6), pp. 835-838.

George, L. a. T. P., 2001. Increasing Physics Enrolment in Year 11. Sydney, New South Wales, Australia, s.n.

Gross, J. L., 2002. Seeing is believing: classroom demonstrations as scientific inquiry. J Phys Teach Educ

Online, Volume 1, pp. 3-6.

Hamer, F. \& Hamer, J., 2004. The potter's dictionary of materials and techniques. s.I.:University of.

Institute of Physics, n.d. Gamma cameras. [Online]

Available at: http://www.iop.org/education/teacher/resources/teaching-medical-

physics/gamma/page 54689.html

[Accessed 7 July 2016].

Johnson, D. W., Johnson, R. T. \& Smith, K. A., 1998. Cooperative learning returns to college what. Change: the magazine of higher learning, 30(4), pp. 26-35.

Jørgensen, K. D. \& and Posneb, A. S., 1959. Study of the setting of plaster. Journal of dental, 38(3), pp. 491-499.

Laukenmann, M. et al., 2003. An investigation of the influence of emotional factors on learning in physics. International Journal of Science Education, 25(4), pp. 489-507.

Lavalette, R. P. M. H. \& Dickstein, H., 1982. Setting temperatures of plaster casts. The. Bone Joint Surg Am, 64(6), pp. 907-911.

Leeder, J. D., 1984. Wool: nature's wonder fibre. Victoria, Australia: Australasian Textiles Publishers. Murphy, P. a. W. E., 2006. Girls and physics: Continuing barriers to 'belonging'.. The Curriculum Journal, 17(3), pp. 281-305.

Peyton, J., 1998. Teaching and learning in medical practice. Rickmansworth: Manticore Europe.

Radiological Society of North America, 2016. RadiologyInfo. [Online]

Available at: http://www.radiologyinfo.org/en/info.cfm?pg=gennuclear

[Accessed 7 July 2016].

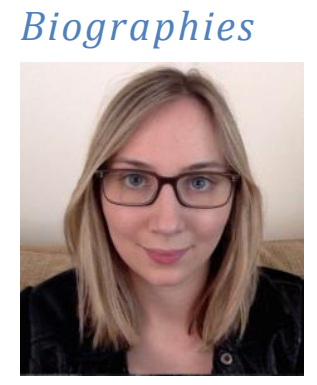

Nicola Wolff is studying Medicine at University College London, and has just completed a Medical Physics and Biomedical Engineering intercalated B.Sc. She developed 'Gamma Anna' during her final year project. 


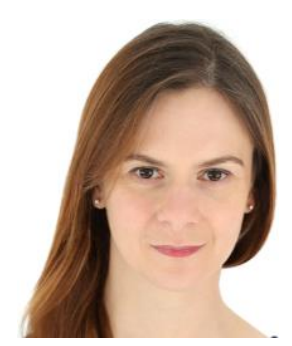

Jennifer Griffiths is a Principal Teaching Fellow at University College London, where she is Director of the Engineering Postgraduate Taught Transformation Programme. Since graduating with a PhD in Medical Radiation Physics in 2005, she has worked in Radiation Physics research, European project management and university teaching. Jenny has a passion for outreach, her activities ranging from object based show and tells at primary schools, through performing at Bright Club, to an in-depth discussion of radiotherapy with a University of the Third Age interest group.

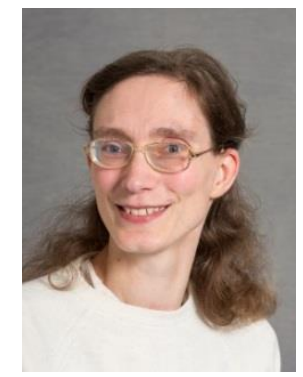

Rebecca Yerworth received her MRes degree in advanced instrumentation studies in 1996 and her PhD, about fluid flow in incontinence pads in 2000, both from University College London. She obtained a PGCE in 2008 on post compulsory education, from the Institute of Education, London. After periods in postgraduate research and teaching, she is now a Senior Teaching Fellow at University College London, working on the Biomedical Engineering degree program. Her research area is Electrical Impedance Tomography. 